\title{
Potential of Stimuli-Responsive Star Polymer for Cancer Targeting
}

\section{Patihul Husni $^{1,2 *}$, M Alvien Ghifari ${ }^{3}$, Norisca Aliza Putriana ${ }^{1}$}

${ }^{1}$ Department of Pharmaceutics and Pharmaceutical Technology, Faculty of Pharmacy, Universitas Padjadjaran, Jatinangor 45363, Indonesia

${ }^{2}$ Department of Global Innovative Drug, College of Pharmacy, Chung-Ang University, 221 Heukseok dong, Dongjak-gu, Seoul 06974, Korea

${ }^{3}$ Department of Chemistry, Faculty of Science, Institut Teknologi Sumatera, Lampung Selatan, 35365, Indonesia

Received : 27 Okt 2020, Revised : 30 Nov 2020, Accepted : 3 Des 2020, Published : 10 Dec 2020

\begin{abstract}
Application of stimuli-responsive star polymers in cancer targeting and drug delivery has been extensively researched because of their several advantages in comparison with their linear counterparts. Functionalization and recombination of various arm architectures of the star polymer are very possible to be conducted to match with numerous needs. The star polymers could not only load more therapeutic drug due to more arms than linear polymers but also be functionalized with targeted moieties for more targeted delivery. Furthermore, the chains in star polymers could be regulated to produce stimuli-responsive star polymer for cancer targeting. This review article aimed to describe the benefits of star polymers and the types of stimuli-responsive delivery system for cancer targeting. Over the last decade, stimuli-responsive star polymers for cancer targeting using either internal stimuli (e.g., $\mathrm{pH}$, redox, enzyme, hypoxia) or external stimuli (e.g., thermal, ultrasound, light, magnetic) has garnered immense interest for researchers. Possibility to mimic a complex natural phenomenon could be achieved by incorporating various stimuli-responsive functionalities in the star polymer.
\end{abstract}

Keywords: stimuli responsive; star polymer; cancer, drug delivery

\section{Introduction}

To date, cancer is still top trigger of the global death. In 2018, cancer is responsible for an estimated 9.6 million deaths (1). It is predicted that $>20$ million new cancer case annually expected by 2025 (2). World Health Organization (WHO) estimates the continue rising of deaths to 11.5 million by 2030 worldwide (3). Cancer can spread into or invade tissue. Cancer cell can spread or invaded tissue by breaking off the cell and travelling to other places through blood or lymph system. As the result new tumors are formed (4). Currently, researchers around the world have put their effort to obtain newer and more potent drug delivery systems (DDSs) to treat cancer. The DDSs may overcome the problem such as unfavorable biodistribution and rapid clearance from the body (5). The DDS should be designed to deliver cancer drug to cancerous cells only and not to surrounding healthy tissue. The concentration of cancer drug must increase specifically at the tumor site resulting in high treatment efficacy with low side effects $(5,6)$.

Many DDSs have been developed to target cancerous cells over the years. In addition, many different strategies have been evaluated such as passive targeting, active targeting to cancer cells or endothelial cells and triggered drug delivery (5). Effective carriers are crucial to develop, to realize successful therapies in cancer treatment. The effectiveness of the carriers can be defined by 
their nontoxicity, ability to carry anticancer molecule, which targeted specific cells, and combination therapy property (7). Using sitespecific DDSs, drug can achieve tumor in therapeutic concentration for a continual period of time with low systemic toxicity (8). The targeted drug delivery is mainly used to control the distribution of active molecule and control delivery system based on polymer (7). The use of DDSs based on polymer has been widely investigated and many studies reported the tailoring polymer architecture or composition and evaluation the physicochemical, in vitro and in vivo properties of the polymers (9-13). In recent years, polymeric materials have gained a lot of attraction in DDSs due to its effectiveness in cancer therapy (14-16). Advancement in polymer engineering have led to the fabrication of complex star polymer architectures with unique properties and applications that can be synthesized easily (17).

Star polymers can be grouped into a class of branched macromolecules with adjustable several linear chains radiating from a core (Figure 1). Many studies report advantages of star polymers compared to linear polymers (18-23).

Interestingly, the role of star polymers in drug delivery and cancer targeting are that (a) star polymers possess an ability to selfassembled into a massive structure which can be designed using their functionalizable arms. This unique property cannot be met by the simple linear polymer. In addition, (b) as their designable architecture, modifying the arm to control biodegradation rate is very possible. Moreover, (c) star polymer can be incorporated with stimuli responsive moiety for DDSs and synthesized with certain biomarkers to target cancer cells. The star polymers mainly useful to deliver hydrophobic molecules in aqueous environment (17). The star polymers with more arms can not only load more drug molecules than their linear counterparts but also be functionalized with targeted molecules for more targeted delivery (18-21, 24, 25). In star polymers, the linear branching chains can be chemically modulated to fabricate stimuli-responsive materials (17). Stimuli-responsive materials are ideal for applications in drug DDSs to target cancer cells because they are capable of automatically undergoing conformational or chemical changes upon receiving stimulus from the environment (17). Some typical signals include changes in $\mathrm{pH}$, temperature, redox trigger substance, enzyme, oxygen level causing hypoxia, irradiation with light, exposure to ultrasound or magnetic field (26). The responses resulted by these stimuli are a change in structural or shape, chain conformation, solubility, surface activity, color or transparency, increased permeability to water, sol-gel transition and so forth $(7,26-$ 28). The stimuli-responsive functions facilitate drug release to increase drug delivery specificity, efficacy and biological activities (26).

Application of stimuli-responsive star polymers in cancer targeting and drug delivery has been extensively studied by researcher. For example, Li et al. prepared 4-armed star copolymer of tetra-(methoxy-poly(ethylene glycol)-poly(2-(N,N-diethylamino)ethyl methacrylate)-poly( $\varepsilon$-caprolactone) pentaerythritol as a $\mathrm{pH}$-responsive nanocarrier to deliver anticancer drugs for cancer targeting (29).
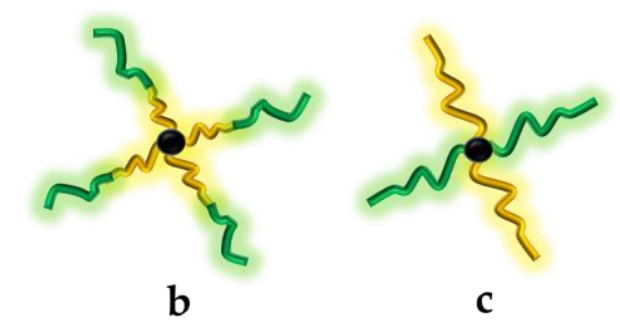

Figure 1. Description of (a) star-shaped polymer, (b) star-shaped block copolymer, and (c) miktoarm star polymer 
In addition, redox-sensitive star polymer consisted of poly( $\varepsilon$-caprolactone)poly(ethylene glycol) for targeted anticancer drug delivery was synthesized by Shi et al (30). Other researchers developed multistimuli-responsive star polymer with more than one responsive moiety for cancer targeting using thermal and $\mathrm{pH}$ sensitive material (31), light, $\mathrm{pH}$ and redox material (32) or other combination of responsive materials. In this review, we focus on the benefits of star polymers and the types of stimuli-responsive delivery system for cancer targeting.

\section{Benefits of Star Polymers}

Linear polymers are non branched or intramolecular bridges of polymer chain but the structural unit is in a single line (33). In contrast, star polymers are composed of several linear chains which is linked to central point or core (34) (Figure 1). In comparison to linear chain polymer, the star structure offers more advantages, such as those in Table 1.

Table 1. Benefits of star polymers.

\begin{tabular}{cc}
\hline Parameters & References \\
\hline Lower critical micelle & $(18)$ \\
concentration $(\mathrm{CMC})$ & $(18,19)$ \\
Higher stability & $(18-21)$ \\
Higher drug loading content & $(22,23)$ \\
and drug loading efficiency & Smaller micelle size \\
Smaller hydrodynamic \\
diameter
\end{tabular}

A CMC value is defined as parameter related to micellization ability and stability of micelle. The smaller CMC value, the stronger the micelle-forming ability and micelle stability. Original morphology of the micelle can be retained by a lower CMC value of the polymer until the micelles reach the target site. Extreme dilution of the micelles can occur when intravenous injection is given to human. While the micelles circulate in the blood stream below or above its CMC value, the stable micelles have slower dissociation so it can retain their integrity and drug content (35). A low CMC value of star polymers can be attributed to the hydrophobic interaction of the polymer and the length of the hydrophobic segments (36). The specific benefit of the low $\mathrm{CMC}$ value of the star polymer on the delivery system for cancer targeting is that a low CMC value of the polymer may achieve greater accumulation in the cancer cells because a lower CMC value indicates improved structure stability during systemic circulation until the carrier reach the cancer cells (37).

Stability of star polymer-based unimolecular micelles is higher than micelles from amphiphilic molecules due to covalently fixed branching points of unimolecular micelles (the lipophilic components are covalently bound together) $(35,38)$. In addition, the main reason probably is that the star polymers have intrinsic core-shell structures and the hyperbranched core structures plays a role to reduce the risk of micellar disassembly effectively (18).

Polymer architecture greatly influences drug encapsulation. This drug encapsulation is affected by solvent type, concentration and duration when loading drugs process. The drug encapsulation mainly depends on the affinity of intrinsic interaction between drug and certain groups of hydrophobic segment (35). Higher drug loading of poorly water soluble or hydrophobic drug is caused by the increased or higher hydrophobicity of polymer block $(39,40)$. The star polymers provide large cavities to load more hydrophobic drug (18). Moreover, increasing the length of polymer chain and subsequently the molar mass of star polymers have substantial effect on material's loading capabilities (20). Star polymers have more arms which able to carry more drug molecules than linear polymers (19).

The micelle size is also critical factor for drug delivery. Delivery systems that are smaller than $200 \mathrm{~nm}$ provide long circulation time of micelles in the blood, reduce uptake by the reticuloendothelial system (RES), and facilitate the extravasation at leaky site of capillaries. Renal elimination can be prevented if the micelles have high molecular weight (> $\left.10^{6} \mathrm{~g} / \mathrm{mol}\right)$. Meanwhile, supramolecular structures often accumulate in the spleen and 
liver because of their large size or protein adsorption which trigger rapid uptake by RES (35). Star polymers have smaller micelle size than for linear copolymer due to the more compact nature of star polymers (41).

The constrained geometry architecture and the molecular interactions among the star polymers lead to a smaller hydrodynamic volume. The smaller hydrodynamic size will have higher cellular uptake efficiency (21).

\section{Types of Stimuli Responsive Delivery System for Cancer Targeting}

The role of stimuli-based delivery system is a critical point in DDSs because of the environmental variation in cancer cells. Interestingly, responsive materials are able to mimic some biological processes and recognize at the molecular level to manipulate development of custom-designed molecules to increase the specificity of cancer drug delivery and for targeting cancer cells $(26,42)$. The responsive materials respond to different stimuli or changes in the environment (7). Stimuli-responsive DDSs for cancer targeting divide into external stimuli and internal stimuli (Figure 2). In case of internal stimuli, the internal triggers are intrinsically existed in tumor microenvironment or inside cancer cells. In contrast to internal stimuli, externally triggers are applied outer of tumor or cancer cells when the external stimuli method is used (26).

Internal stimuli can be categorized as $\mathrm{pH}$, redox, enzyme, and hypoxia. $\mathrm{pH}-$ responsive polymers contain weakly acidic or basic ionizable groups (pKa values 3 - 10) which either donate or accept protons in response to changes in environmental $\mathrm{pH}$. A change in ionization state as function of $\mathrm{pH}$ resulted alteration in structural and other properties (chain conformation, solubility, surface activity, etc.) is demonstrated by the acidic or basic groups of polyelectrolytes (carboxylate, sulfonate, and amino groups). The $\mathrm{pH}$-responsive polymers have different characteristic under a neutral and acidic or basic condition. Electrostatic repulsion occurs between charged groups as a result an increase in degree of ionization (7). In case of redoxresponsive delivery system, the glutathione (GSH) level inside cancer cells $(2-10 \mathrm{mM})$ is remarkable higher than that in normal regions $(2-10 \mu \mathrm{M})(26)$. Hence, the GSH presence in higher concentration within tumor or the microenvironment of the cancer cells. The GSH acts as a trigger for the reduction of the disulfide bond (SS) linkage. The redoxresponsive function could trigger the dissociation and degradation carriers inside cancer cells (30). Enzyme-

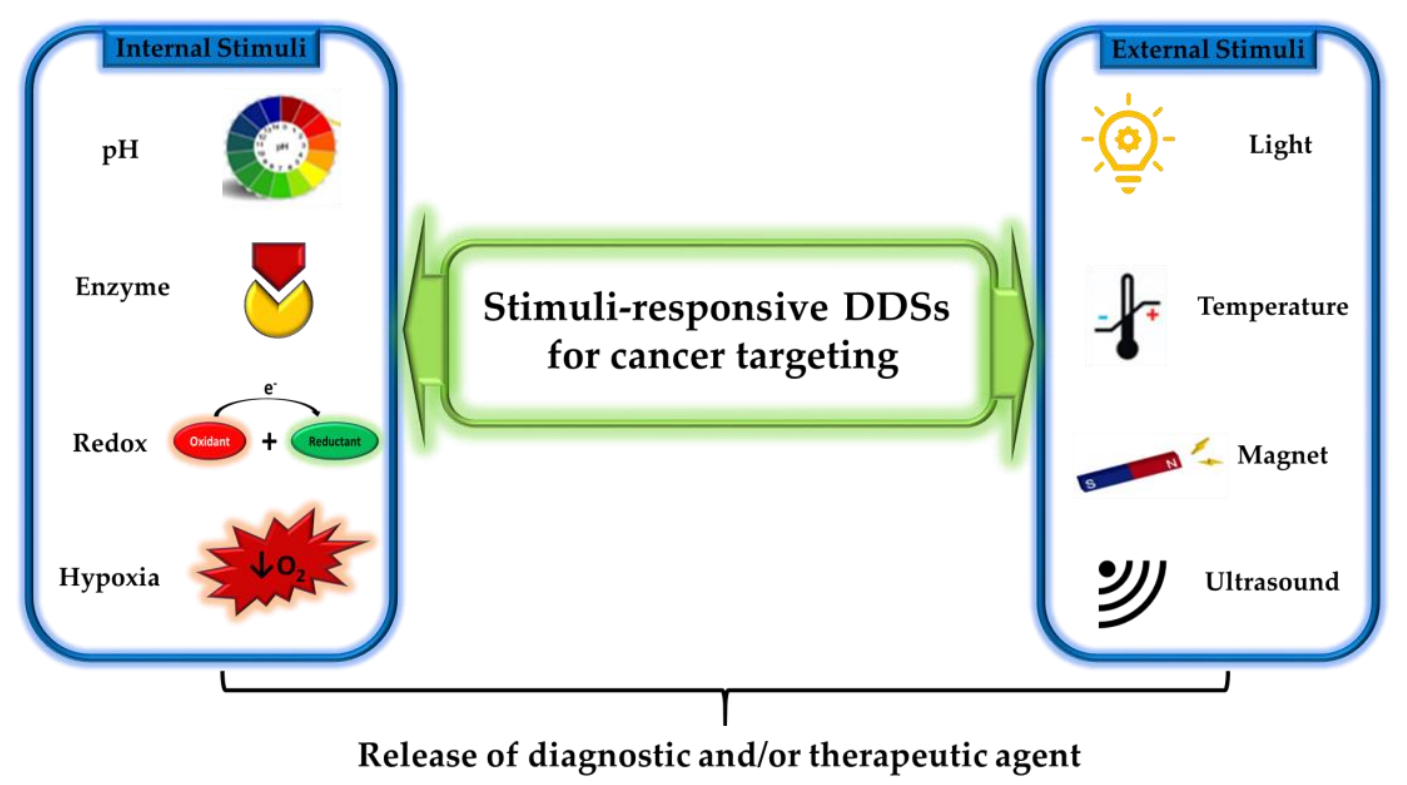

Figure 2. Description of types of stimuli-responsive DDSs used for cancer targeting 
responsive delivery systems are based on the response to several upregulated enzymes in tumor microenvironment and cancer cells (26). There is a changed articulation of catalyst induced by enzyme observed in cancer cells (42). Over-expression of enzymes by tumor cells is resulted for tumor growth, angiogenesis, invasion and metastasis (43). In relation to hypoxia-responsive delivery system, hypoxia could trigger cargo release from the hypoxia-sensitive carriers due to the cleavage of hypoxia-sensitive cross-linkers. Tumor hypoxia (low oxygen level) is likely caused by poorly vascularization inside solid tumors (26). An over consumption of oxygen by rapidly proliferating cells of tumor results in tumor hypoxia and also due to inconsistent flux of erythrocyte in the abnormal tumor vasculature (43).

External stimuli can be triggered by thermal, ultrasound, light and magnetic. Thermal-triggered delivery systems are generally designed to change significantly in their properties by responding to the narrow temperature shift. The thermal-responsive carriers are stable in temperature up to $37^{\circ} \mathrm{C}$ and sensitive to higher temperature $\left(>40^{\circ} \mathrm{C}\right)$ for achieving thermal-triggered drug release and therapy (26). To utilize the ultrasound, a high frequency sound waves are needed. The sound wave above $20 \mathrm{kHz}$ are applied either to disrupt carrier to release the cargo or enhance the cell membrane's permeability. Other than that, the light irradiation (UV-Vis and nearinfrared light) generally could remotely affect the light-responsive carriers in cancer cells (26). The optical signal induces the photochromic molecule (chromophores) in the photoreceptor to convert the light-irradiation into a chemical signal by photoreaction process. For magnetic-responsive delivery system, generally, strategy used in developing magnetic-responsive delivery system is either incorporating magnetic materials into carriers for achieving magnetic-sensitivity or locating a permanent magnetic field in malignant tissue after administration.

Majority of the delivery system based on stimuli-responsive deals with response to a single stimulus. However, biological performance of macromolecules shows changes in response to a combination of stimuli. In order to mimic biological processes, different stimuli-responsive functionalities can be incorporated in a single polymer to create multi-stimuli-responsive materials to provide more than one mechanism responsiveness for targeting cancer cells (26, 42). Star polymer for drug delivery to cancer targeting seems to be important candidate because of their unique properties.

\section{Conclusion}

Stimuli-responsive materials have received increasing interest in recent years, due to its unique properties. The star polymers can load more drug molecules and be functionalized with targeted molecules for more targeted delivery. The responsive materials respond to different stimuli or changes in the cancer environment. Until now, numerous internal and external stimuliresponsive star polymers have been developed to mimic some biological processes, recognize at molecular level and facilitate drug release to increase drug delivery specificity, efficacy and biological activities for cancer targeting.

\section{References}

1. Organization WH. Cancer Geneva, Switzerland: (WHO); 2018 [Available from: https://www.who.int/en/newsroom/fact-sheets/detail/cancer.

2. Zugazagoitia J, Guedes C, Ponce S, Ferrer I, Molina-Pinelo S, Paz-Ares L. Current challenges in cancer treatment. Clinical Therapeutics. 2016;38(7):155166.

3. Organization WH. Cancers: WHO; 2009 [Available

from: https://www.who.int/nmh/publications/f act_sheet_cancers_en.pdf.

4. Institute NC. What is cancer? : National Cancer Institute; 2015 [Available from: https://www.cancer.gov/aboutcancer/understanding/what-is-cancer.

5. Lammers T, Kiessling F, Hennink WE, Storm G. Drug targeting to tumors: 
principles, pitfalls and (pre-) clinical progress. Journal of Controlled Release. 2012;161(2):175-87.

6. Raavé R, van Kuppevelt TH, Daamen WF. Chemotherapeutic drug delivery by tumoral extracellular matrix targeting. Journal of Controlled Release. 2018;274:1-8.

7. Alsuraifi A, Curtis A, Lamprou DA, Hoskins C. Stimuli responsive polymeric systems for cancer therapy. Pharmaceutics. 2018;10(3):136.

8. Wang F, Saidel GM, Gao J. A mechanistic model of controlled drug release from polymer millirods: effects of excipients and complex binding. Journal of Controlled Release. 2007;119(1):111-20.

9. Rabanel J-M, Faivre J, Paka GD, Ramassamy C, Hildgen P, Banquy $\mathrm{X}$. Effect of polymer architecture on curcumin encapsulation and release from PEGylated polymer nanoparticles: Toward a drug delivery nano-platform to the CNS. European Journal of Pharmaceutics and Biopharmaceutics. 2015;96:409-20.

10. Liang Y, Deng X, Zhang L, Peng X, Gao W, Cao J, et al. Terminal modification of polymeric micelles with $\pi$-conjugated moieties for efficient anticancer drug delivery. Biomaterials. 2015;71:1-10.

11. Wang Z, Chen C, Zhang Q, Gao M, Zhang J, Kong D, et al. Tuning the architecture of polymeric conjugate to mediate intracellular delivery of pleiotropic curcumin. European Journal of Pharmaceutics and Biopharmaceutics. 2015;90:53-62.

12. Braunová A, Chytil P, Laga R, Š́rová M, Machová D, Parnica J, et al. Polymer nanomedicines based on micelleforming amphiphilic or water-soluble polymer-doxorubicin conjugates: Comparative study of in vitro and in vivo properties related to the polymer carrier structure, composition, and hydrodynamic properties. Journal of Controlled Release. 2020.
13. Ali I, Kareem F, Rahim S, Perveen S, Ahmed S, Shah MR, et al. Architecture based selectivity of Amphiphilic block copolymers of poly (ethylene oxide) and poly ( $\varepsilon$-caprolactone) for drug delivery. Reactive Functional Polymers. 2020:104553.

14. Chen W, Zhou S, Ge L, Wu W, Jiang X. Translatable high drug loading drug delivery systems based on biocompatible polymer nanocarriers. Biomacromolecules. 2018;19(6):173245.

15. Sun X, Wang G, Zhang H, Hu S, Liu X, Tang J, et al. The blood clearance kinetics and pathway of polymeric micelles in cancer drug delivery. ACS Nano. 2018;12(6):6179-92.

16. Fenton OS, Olafson KN, Pillai PS, Mitchell MJ, Langer R. Advances in biomaterials for drug delivery. Advanced Materials. 2018;30(29):1705328.

17. Yang DP, Oo MNNL, Deen GR, Li Z, Loh XJ. Nano-star-shaped polymers for drug delivery applications. Macromolecular Rapid Communications. 2017;38(21):1700410.

18. Zhang S, Hou Y, Chen H, Liao Z, Chen $\mathrm{J}, \mathrm{Xu} \mathrm{BB}$, et al. Reduction-responsive amphiphilic star copolymers with longchain hyperbranched poly $(\varepsilon-$ caprolactone) core and disulfide bonds for trigger release of anticancer drugs. European Polymer Journal. 2018;108:364-72.

19. Chen Y, Yang Z, Liu C, Wang C, Zhao $S$, Yang J, et al. Synthesis, characterization, and evaluation of paclitaxel loaded in six-arm star-shaped poly (lactic-co-glycolic acid). International Journal of Nanomedicine. 2013;8:4315.

20. Byrne M, Thornton PD, Cryan S-A, Heise A. Star polypeptides by NCA polymerisation from dendritic initiators: synthesis and enzyme controlled payload release. Polymer Chemistry. 2012;3(10):2825-31. 
21. Zeng X, Tao W, Mei L, Huang L, Tan C, Feng S-S. Cholic acid-functionalized nanoparticles of star-shaped PLGAvitamin $\mathrm{E}$ TPGS copolymer for docetaxel delivery to cervical cancer. Biomaterials. 2013;34(25):6058-67.

22. Soliman GM, Sharma R, Choi AO, Varshney SK, Winnik FM, Kakkar AK, et al. Tailoring the efficacy of nimodipine drug delivery using nanocarriers based on A2B miktoarm star polymers. Biomaterials. 2010;31(32):8382-92.

23. Aliferis $\mathrm{T}$, Iatrou $\mathrm{H}$. Aggregation phenomena of linear and miktoarm star copolymers of styrene and dimethylsiloxane: Influence of the architecture. European Polymer Journal. 2008;44(7):2412-7.

24. Pan Y, Cai P, Farmahini-Farahani M, Li $Y$, Hou $X$, Xiao H. Aminofunctionalized alkaline clay with cationic star-shaped polymer as adsorbents for removal of $\mathrm{Cr}$ (VI) in aqueous solution. Applied Surface Science. 2016;385:333-40.

25. Azuma $\mathrm{Y}$, Terashima T, Sawamoto M. Precision synthesis of iminefunctionalized reversible microgel star polymers via dynamic covalent crosslinking of hydrogen-bonding block copolymer micelles. Macromolecules. 2017;50(2):587-96.

26. Mi P. Stimuli-responsive nanocarriers for drug delivery, tumor imaging, therapy and theranostics. Theranostics. 2020;10(10):4557.

27. Guragain S, Bastakoti BP, Malgras V, Nakashima K, Yamauchi Y. Multistimuli-responsive polymeric materials. Chemistry-A European Journal. 2015;21(38):13164-74.

28. Schattling $\mathrm{P}$, Jochum FD, Theato P. Multi-stimuli responsive polymers-the all-in-one talents. Polymer Chemistry. 2014;5(1):25-36.

29. Li Q, Jin YG, Li M, Dong JX. DrugLoaded Star-Shaped pH-Responsive Monomolecular Copolymer Nanocarriers for Tumor Targeting and
Cancer Therapy. Acs Biomater Sci Eng. 2015;1(3):175-82.

30. Shi C, Guo X, Qu Q, Tang Z, Wang Y, Zhou S. Actively targeted delivery of anticancer drug to tumor cells by redoxresponsive star-shaped micelles. Biomaterials. 2014;35(30):8711-22.

31. Zhou Z, Li G, Wang N, Guo F, Guo L, Liu X. Synthesis of temperature/pH dual-sensitive supramolecular micelles from $\quad \beta$-cyclodextrin-poly $(\mathrm{N}$ isopropylacrylamide) star polymer for drug delivery. Colloids and Surfaces B: Biointerfaces. 2018;172:136-42.

32. An X, Zhu A, Luo H, Ke H, Chen H, Zhao Y. Rational Design of MultiStimuli-Responsive Nanoparticles for Precise Cancer Therapy. ACS Nano. 2016;10(6):5947-58.

33. Han F, Zhu C, Chen L, Wicks J, Li B. Chapter 3 - Bio-Instructive Scaffolds for Bone Regeneration. In: Brown JL, Kumbar SG, Banik BL, editors. BioInstructive Scaffolds for Musculoskeletal Tissue Engineering and Regenerative Medicine: Academic Press; 2017. p. 55-84.

34. Hadjichristidis N, Pitsikalis M, Iatrou H, Driva P, Sakellariou G, Chatzichristidi MPsacr. 6.03-Polymers with StarRelated Structures: Synthesis, Properties, and Applications. Elsevier: Amsterdam; 2012. p. 29-111.

35. Qiu LY, Bae YH. Polymer architecture and drug delivery. Pharmaceutical Research. 2006;23(1):1-30.

36. Li M, Guo J-W, Wen W-Q, Chen J-K. Biodegradable Redox-Sensitive Star Polymer Nanomicelles for Enhancing Doxorubicin Delivery. Nanomaterials. 2019;9(4):547.

37. Qiu LY, Wang RJ, Zheng C, Jin Y, Jin LQ. $\beta$-cyclodextrin-centered star-shaped amphiphilic polymers for doxorubicin delivery. Nanomedicine. 2010;5(2):193208.

38. Zhu J, Zhou Z, Yang C, Kong D, Wan Y, Wang ZJ. Folate-conjugated amphiphilic star-shaped block copolymers as targeted nanocarriers. 
Journal of Biomedical Materials Research Part A. 2011;97(4):498-508.

39. Crothers M, Zhou Z, Ricardo NM, Yang $\mathrm{Z}$, Taboada P, Chaibundit $\mathrm{C}$, et al. Solubilisation in aqueous micellar solutions of block copoly (oxyalkylene) s. International Journal of Pharmaceutics. 2005;293(1-2):91-100.

40. Zhang X, Jackson JK, Burt HMJjop. Development of amphiphilic diblock copolymers as micellar carriers of taxol. 1996;132(1-2):195-206.
41. Ren JM, McKenzie TG, Fu Q, Wong $\mathrm{EH}, \mathrm{Xu} \mathrm{J}$, An Z, et al. Star polymers. Chemical Reviews. 2016;116(12):6743836.

42. Pethe AM, Yadav KS. Polymers, responsiveness and cancer therapy. Artificial Cells, Nanomedicine, Biotechnology. 2019;47(1):395-405.

43. Fernandes C, Suares D, Yergeri MC. Tumor microenvironment targeted nanotherapy. Frontiers in Pharmacology. 2018;9:1230. 